\title{
Design and Construction of a Sun Simulator for Laboratory Testing of Solar Cells
}

\author{
István Bodnár ${ }^{1}$, Dániel Koós ${ }^{2}$, Patrik Iski ${ }^{3}$, Ádám Skribanek ${ }^{4}$ \\ ${ }_{1,2,3,4}$ University of Miskolc, Faculty of Mechanical Engineering and Informatics, \\ ${ }^{1,3,4}$ Isntitute of Electrical and Electronic Engineering, \\ ${ }^{2}$ Isntitute of Energy Engineering and Chemical Machinery, \\ 12vegybod@uni-miskolc.hu, ${ }^{2}$ elke100@uni-miskolc.hu, ${ }^{3}$ elkvmt@uni-miskolc.hu, \\ ${ }^{4}$ elkskrib@uni-miskolc.hu, ${ }^{1,2,3,4}$ H-3515 Miskolc-Egyetemváros
}

Abstract: The current study is dedicated to the realization and development of an ASTM E972 standardized, class C sunlight simulator. Sunlight simulators or sun simulators are laboratory devices used to study solar cells. Our sun simulator is suitable for the examination and qualification of solar cells, with an area of $150 \mathrm{~mm} \times 150 \mathrm{~mm}$. It consists of 4 LED blocks of different colors and a halogen reflector. The completed sun simulator is validated; the design and realized parameters display good matching.

Keywords: sun simulator; solar simulator; solar cell tester; halogen; LED

\section{Introduction}

Energy production, which inevitably involves pollution, is indispensable in our society. Producing energy in a way that is environmentally-friendly is possible with renewable sources, of which, the source is usually (with some exceptions, such as geothermal energy and nuclear) solar energy related. This energy reaches the surface in the form of radiation, which can be harvested via solar panels, solar thermal collectors and/or solar concentrators.

Today's widespread mono- and polycrystalline solar panels work with an efficiency of $14-18 \%$. Research is continuously being carried out, in order to achieve a higher rate of efficiency and complex investigations of currently used technologies are also required. The quality of illumination of solar panels is a high priority factor in laboratory examinations, which is determined by how similar the facilitated sun simulator's light is compared to natural illumination [5, 8, 21, 24].

A sun simulator is a device that provides illumination, of which, the intensity and spectral composition is similar to that of natural sunlight inside the inspection range. The similarity depends on the light of the facilitated sun simulator. Using 
such equipment, the simulated sunlight is continuously available, relatively homogeneously distributed, hence can be used for various indoor or long-term investigations. Since it is regulable, it is commonly used to inspect solar cells, awnings, plastics and other materials, and sunlight-sensitive devices [5, 21, 24].

Sun simulators usually consist of three main units. Any sun simulator has light sources and power supplies. Optical light filters can be used to achieve appropriate illumination, and the use of a control unit is essential so that the properties of the light can be changed as desired.

First generation sun simulators were generally based on halogen, xenon, or tungsten light sources, and their combinations. To achieve homogeneous light intensity, complex optical systems had to be installed. Acquiring the desired spectral composition required optical filters. In the 1990s, with the rapid advancement of semiconductor technology, new sun simulator types have seen the light. They operated with a cheaper, more compact and more energy efficient alternative of previously mentioned light sources; LED units. These devices can be described as easily regulable, and a huge advantage of LED based light sources is that, by applying different colors, a similar spectral composition to that of natural sunlight can be achieved. Note that by relying solely on LED lights, 1,000 $\mathrm{W} / \mathrm{m}^{2}$ light intensity, which is required for standardized examinations, can hardly be achieved. However, applying a combination of LED lights and conventional illumination can mean the solution to this problem $[1-4,12,25]$.

Our goal is to develop an ASTM E972 standardized [1-4, 12, 25], class C sun simulator, which is suitable to illuminate an inspection area of $150 \mathrm{~mm} \times 150 \mathrm{~mm}$, making the standardized examination of small size solar cells possible.

\section{Design}

This section briefly describes the relevant standards, and details the steps and results of the design process.

\subsection{ASTM E972 Standard}

Requirements considering sun simulators are found in the American Standard for Testing and Materials (ASTM) E972. According to this standard, sun simulators can be classified by three main aspects into different classes, however, any sun simulator must be able to provide the average surface light intensity (AM 1.5) with a maximal $1,000 \mathrm{~W} / \mathrm{m}^{2}$ value $[1-4,25]$.

When inspecting the conformity of a sun simulator with standards, the similarity between the spectral composition of the illumination and natural sunlight is examined, as well as the temporal and spatial unevenness of said illumination. 
The standard states that the spectral conformity in case of AM 1.5 is to be examined between the wave lengths of 400 and 1,100 nm. Sun simulators can then be classified into class A, B or C based on the previously mentioned three aspects. The requirements of the three groups are summarized in Table 1 [1-4, 25].

Table 1

Category requirements for standardized sun simulators

\begin{tabular}{|l|c|c|c|}
\cline { 2 - 4 } \multicolumn{1}{c|}{} & Class A & Class B & Class C \\
\hline Spectral conformity with natural sunlight & $75-125 \%$ & $60-140 \%$ & $40-200 \%$ \\
\hline Spatial unevenness & $\leq 2 \%$ & $\leq 5 \%$ & $\leq 10 \%$ \\
\hline Temporal unevenness & $\leq 2 \%$ & $\leq 5 \%$ & $\leq 10 \%$ \\
\hline
\end{tabular}

The stricter requirements of class A and B sun simulators carry a higher cost and an increased time of development, hence taking the feasibility into account, we set the development of an ASTM E972 standardized, class C sun simulator as the main objective for our research and development project. For future research projects with small size solar cells, said class $\mathrm{C}$ simulator provides a suitable laboratory light source, as the solar intensity to be provided is the same for every class. For a class $\mathrm{C}$ sun simulator, however, the deviation of spectral conformity, and its spatial and temporal unevenness hardly affect the final measurement results, therefore the use of a higher-class sun simulator is unnecessary to successfully carry out the measurements $[1-4,25]$.

\subsection{Design Parameters, Construction Basics}

Our goal is to design a class $\mathrm{C}$ sun simulator which complies the ASTM E972 standard. The sun simulator consists of a combination of high-performance, colored LED units and halogen lamps; by implementing LED units with appropriate colors, a similar spectral composition to that of natural sunlight can be achieved, that is between the wave lengths of 400 and $700 \mathrm{~nm}$. But relying solely on LED lights, the $1,000 \mathrm{~W} / \mathrm{m}^{2}$ light intensity required for standardized examinations can - in this case - not be achieved, hence, the application of halogen lamps. Further advantages of the concept are that by arranging the LED units in a matrix form, a relatively homogeneous light intensity distribution can be achieved, and the spectral distribution of the halogen lamp is similar to that of the sunlight within the wavelength range of 700 and 1,100 $\mathrm{nm}[1-4,9,12,14,25]$.

After laying the foundations of the construction, it is now necessary to define a number of parameters which are going to significantly influence future results.

In our case, we can distinguish fixed parameters and so-called design parameters. Fixed parameters are set based on previous measurements or considerations, while the optimal values of the design parameters will be determined in a later phase of the design process. Fixed parameters are summarized in Table 2. 
Table 2

Fixed parameters of LED units, halogen light sources, and the inspection range

\begin{tabular}{|l|l|}
\hline \multicolumn{2}{|c|}{ LED } \\
\hline Number & $\begin{array}{l}36 \text { pieces } \\
(6 \times 6 \text { matrix arrangement })\end{array}$ \\
\hline Colours & neutral white, red, green, blue \\
\hline Distance between LED units in the matrix & $3 \mathrm{~cm}$ \\
\hline Maximal performance of LED units & $3 \mathrm{~W}$ \\
\hline \multicolumn{2}{|c|}{ Halogen lamps } \\
\hline Maximal performance of halogen lamps & $50 \mathrm{~W}$ \\
\hline Placements of halogen lamps & $\begin{array}{l}\text { at the corners and/or sides of the LED } \\
\text { matrix }\end{array}$ \\
\hline Number of halogen lamps & $2 \times 4=8$ pieces \\
\hline \multicolumn{2}{|c|}{ Inspection range } \\
\hline Size of inspection range & $15 \mathrm{~cm} \times 15 \mathrm{~cm}$ \\
\hline Distance from the LED matrix & $8 \mathrm{~cm}$ \\
\hline
\end{tabular}

The size of the inspection range is determined by the size of the solar cells, which is usually $15 \mathrm{~cm} \times 15 \mathrm{~cm}$. The number of LED units can be determined by considering that the size of the matrix is slightly larger than the size of the inspection range, but it is also necessary for the final number of LEDs to be a multiple of four, since four LED colors are implemented. The main considerations when choosing the colors of the LED units are the availability and the supposed spectral composition of individual colors. The available color palette is as follows: neutral white, cold white, orange, red, green, blue. The cold white spectrum is similar to the neutral white, but it has a dominant blue vibe, which becomes dispensable by implementing the blue as an individual color. The spectrum of orange highly overlaps with red, hence using both at the same time is superfluous. Accordingly, neutral white, red, green, and blue are chosen.

The distance between the LED matrix and the inspection range is chosen to be as short as possible to maximize light intensity, but taking the halogen lamps into account as well, which due to their size require a larger space, $8 \mathrm{~cm}$ distance between the two units is necessary. Early in the design, the question of the adjustability of light intensity of the halogen lamps arose. Therefore, a high performance ( $50 \mathrm{~W}$, GU10 socket) halogen lamp seems to be the most expedient choice, giving us the opportunity to adjust the light intensity on a wide scale.

Based on preliminary light intensity measurements, we found that 4 pieces of 50 $\mathrm{W}$ halogen lamps may not be enough to provide the necessary $1,000 \mathrm{~W} / \mathrm{m}^{2}$ light intensity. The number of halogen bulbs is 4 or 8 pieces (which will further be unfolded later in the optimization part). Keeping the symmetry in mind, halogen lamps can be placed in the corners and/or on the sides. 
Contrary to the fixed parameters, some variable parameters require a more detailed design. For LED units, such parameters are the supply amperage of individual color groups. In case of the halogen lamps, we are looking for the optimal number (4 or 8 units) and their positions (horizontal inclination, horizontal and vertical distance from the inspection range).

\subsection{Designing for Spectral Conformity}

One criterion required by the standard, is the spectral conformity of the illumination with sunlight. When designing for spectral conformity, the first step is to determine the spectrum curves of the light sources. However, during the design process we do not have access to a spectrometer to perform an absolute spectral assay, so the spectrum curves are obtained indirectly. Calculations are based on light intensity test measurements, calculations and evaluations are done in MATLAB environment. (The MATLAB code can be found at: http://www.unimiskolc.hu/ vegybod/sunsimulator.m).

In case of the LED units, the spectrum curves can be approximated by Gaussian curves. For this, three practically chosen wavelength - light intensity points are necessary, to which the desired curve will be approximated by the MATLAB code. Similar algorithms are used by Hussain et al. [25]. For LED units, the wavelength values of emission peaks for each colour are given by the manufacturer, and for each colour, the wavelength range in which the emission is still significant is also known. Therefore, we have the necessary points. Hereupon, the relative spectrum curve becomes obtainable for the given colour, although for design purposes the absolute spectral distribution is necessary. This calibration problem can be resolved by considering that the integral of the absolute spectrum curve of the given light source is approximately the same as the value of light intensity it produces. So, if the light intensity and amperage of the different LED colours are known for a particular arrangement, then all the information that is necessary to determine the absolute spectrum curve is given.

In case of halogen lamps, the absolute spectrum structure can be derived from the black-body radiation, assuming a color temperature of $3,200 \mathrm{~K}$ using equation 1 $[5-8,14,16,23]$.

$$
E(\lambda, T)=\frac{2 \pi h c^{2}}{\lambda^{5}\left(e^{\frac{h c}{\lambda k T}}-1\right)}
$$

where: $E-$ spectral intensity

$\lambda$ - wavelength of light

$\mathrm{T}$ - color temperature

$\mathrm{h}$ - Boltzmann-constant

$\mathrm{c}-$ the velocity of light in vacuum 
The superposition of the spectrum curves of individual light sources gives the specific spectrum structure of the sun-simulator for the given variables. The next task is to determine the amperage that can provide the best possible spectral conformity and complies the standard to power the LED units. The spectral conformity is calculated by equation 2 - in case of AM 1.5 global emission - in the wavelength range $400-1,100 \mathrm{~nm}[5-8,14,16,23]$.

$$
S E\left(\lambda_{a}-\lambda_{f}\right)=\frac{\int_{\lambda_{a}}^{\lambda_{f}} E_{N S z}(\lambda) d \lambda}{\int_{\lambda_{a}}^{\lambda_{f}} E_{A M 1,5}(\lambda) d \lambda}
$$

where: $\operatorname{SE}\left(\lambda_{\mathrm{a}}-\lambda_{\mathrm{f}}\right)-$ Spectral difference for the given wavelength range,

$\lambda_{\mathrm{a}}-$ Lower limit of the wavelength range

$\lambda_{\mathrm{f}}$ - Upper limit of the wavelength range

$\mathrm{E}_{\mathrm{NSz}}-$ Spectral intensity of the sun simulator

$\mathrm{E}_{\mathrm{AM} 1.5}-$ Spectral intensity of the sun

The maximum amperage capacity of the $3 \mathrm{~W}$ LED modules is $700 \mathrm{~mA}$.

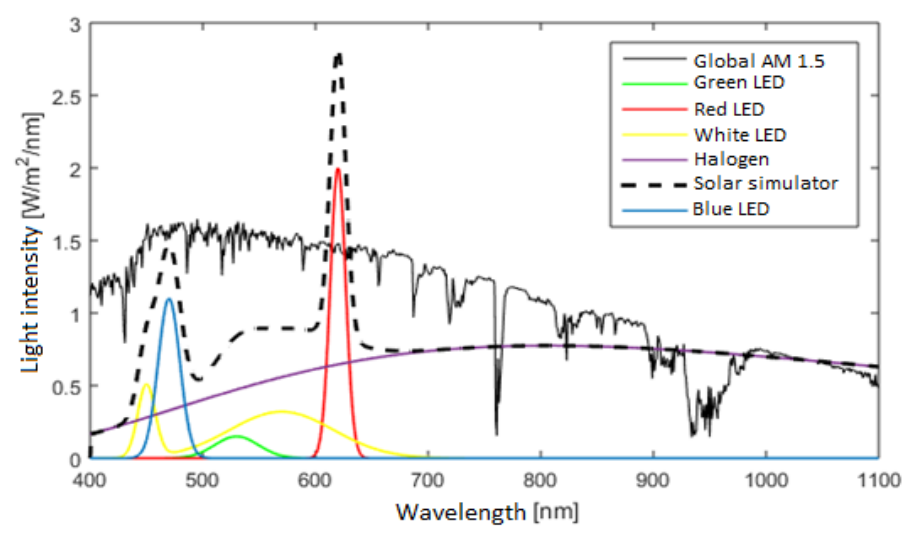

Figure 1

Spectral composition in case of the best matching LED-halogen combination

Five cases with different amperage values are investigated for every color by the MATLAB program we have written, starting from $700 \mathrm{~mA}$ decreasing by $100 \mathrm{~mA}$ steps. For the four colors, this means $5^{4}=625$ combinations in total. Thus, the calculation is executed for 625 amperage combinations; individual spectrum curves and, after the superposition, the specific spectrum structure of the sunsimulator is determined, and the spectral conformity is calculated in every case. The LED power supply combination with the best matching spectrum conformity 
can be selected from the results of the iterations [1-4, 16, 25-29]. The spectral compositions of individual light sources, the sun simulator, and natural sunlight in case of the best matching amperage combination are shown in Figure $1[5-8,14$, $16]$.

\subsection{Designing for Light Intensity Distribution}

Prior to the proper design of the light intensity distribution, test measurements take place. The light intensity distribution generated by the LED and the halogen illumination is calculated separately, and their superposition gives the light intensity distribution produced by the sun simulator. For LED units, measurements are made with the power amperage values used for spectral matching.

The test range is divided into $3 \mathrm{~cm} \times 3 \mathrm{~cm}$ squares and the light intensity measurement is performed in each square. Such a measurement results the light intensity distribution generated by a single LED unit for the given amperage. The expected light intensity distribution is calculated in MATLAB for the array of LED units' matrix, and the calculation is run for 625 amperage combinations [16]. Regarding the halogen lamps, variable parameters are their number (either 4 or 8 ) and their positions. Tests of 30 combinations of halogen position - number pairs are made. The halogen lamp can be positioned at the corners or at the sides of the test range, similarly to the previous one, and the light intensity measurement is performed again in each small square. The result is a light intensity distribution formed by a single halogen lamp at a given position, from which the future light intensity distribution will be determined. Figure 2 illustrates the light intensity distribution obtained by LED and halogen light sources [17-19].
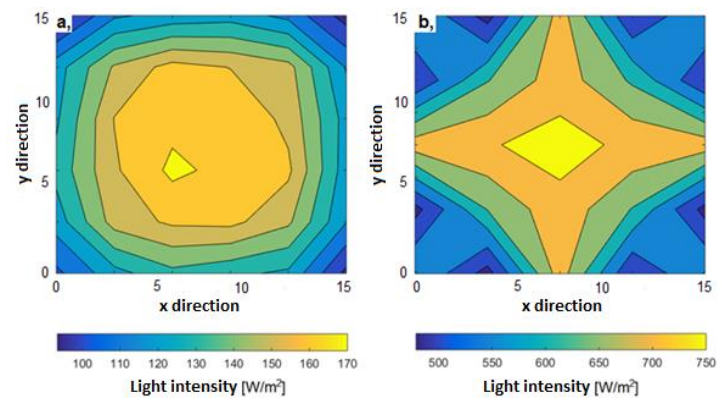

Figure 2

Light intensity distribution of (a) LED matrix, (b) halogen lamps

After the superposition of light intensity distributions produced by the LED and halogen lamps, the next step is to find the variables, with which the inhomogeneity of the light distribution is at nadir. According to the correlation 
given by the standard, the inhomogeneity of the light distribution can be calculated by:

$T_{E g y}=\frac{\left(I_{\max }-I_{\min }\right)}{\left(I_{\max }+I_{\min }\right)} \cdot 100 \%$

where: $\mathrm{T}_{\mathrm{Egy}}-$ unevenness of light intensity

$\mathrm{E}_{\max }-$ maximal light intensity

$\mathrm{E}_{\min }-$ minimal light intensity

The upcoming process is similar to the method used to obtain the spectral distribution. The expected light intensity distribution is calculated for the 625 amperage and 30 position combinations by the MATLAB program, then the inhomogeneity of the current distribution is calculated.

The variable parameters that provide the highest homogeneity can be selected from the results of individual iterations. Figure 3 shows the light intensity distribution with the highest homogeneity. (Note: changing the light intensity of LEDs slightly affects the final light intensity distribution, the positioning of the halogen lamps has a much stronger impact.)

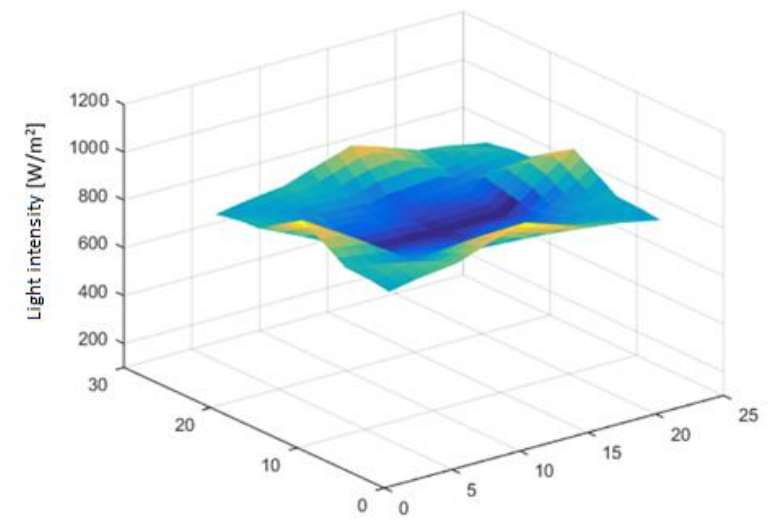

Figure 3

Light intensity distribution with the highest homogeneity inside the inspection range

\subsection{Results of the Design Process}

The purpose of the design method is to determine the optimal values of the previously described variable parameters (the power supply amperage of LEDs, quantity and position of halogen lamps), and to outline the actual design of the device to be constructed. Our designed sun simulator consists of a combination of 36 pieces of colored, $3 \mathrm{~W}$ LED lights, and 8 pieces of $50 \mathrm{~W}$ halogen lamps. Table 3 summarizes the power input of each LED colors separately. (When determining 
these parameters, both spectral conformity and proper light intensity distribution are taken into account.) Table 4 summarizes the ideal positions of the halogen lamps.

Table 3

Power input values for different LED colors

\begin{tabular}{|c|c|c|c|c|c|}
\hline Color & I [mA] & U [V] & Color & I [mA] & U [V] \\
\hline Red & 710 & 2.5 & Green & 670 & 3.2 \\
\hline Blue & 710 & 3.4 & White & 680 & 4.0 \\
\hline
\end{tabular}

Table 4

Planned positions of the halogen lamps

\begin{tabular}{|l|l|l|}
\hline Horizontal distance from the LED matrix & Corner: $30 \mathrm{~mm}$ & Side: $34 \mathrm{~mm}$ \\
\hline Horizontal inclination & Corner: $15^{\circ}$ & Side: $45^{\circ}$ \\
\hline Vertical distance from the inspection range & Corner: $75 \mathrm{~mm}$ & Side: $60 \mathrm{~mm}$ \\
\hline
\end{tabular}

The effectiveness of the design phase can be evaluated according to compliance to standard. In the case of optimum variable parameters, the inhomogeneity of the projected sun simulator's light intensity is $12 \%$ based on the calculations and the average light intensity distribution at this time is $910 \mathrm{~W} / \mathrm{m}^{2}$. The degree of spectral matching in each wavelength range is given in Table 5.

Table 5

Calculated spectral conformity within the inspection range [5-8]

\begin{tabular}{|c|c|c|c|c|c|c|}
\hline Wavelength range & $0.4-0.5$ & $0.5-0.6$ & $0.6-0.7$ & $0.7-0.8$ & $0.8-0.9$ & $0.9-1.1$ \\
\hline $\begin{array}{c}\text { Spectral } \\
\text { conformity }\end{array}$ & 12.8 & 67.0 & 82.3 & 67.9 & 81.5 & 115.3 \\
\hline
\end{tabular}

According to the standard conformity, the following conclusions can be drawn:

- Light intensity and the homogeneity of the light is slightly below of what is specified by the standard. This problem can likely be solved by further optimizing the positions of the halogen lamps on the physical framework.

- As for spectral conformity, the illumination meets the requirements of the class C standard at every wavelength range, except for the $400 \mathrm{~nm}$ to $500 \mathrm{~nm}$ interval. This result suggests the necessity to incorporate a new, low-wavelength dominant (ultraviolet) LED unit.

\section{Construction and Realization}

This chapter describes the construction of the sun simulator with the previously detailed parameters. 


\subsection{Power Supply}

Power supply can be divided into two groups, since the power supply of the halogen lamps and the LED units must both be insured. Powering the halogen lamps is rather simple, since the 8 halogen lamps (with GU10 socket) are powered from the electrical grid through two dimmer switches [12, 17-19]. The halogen lamps can further be divided into two groups according to their dimming; these groups are halogen lamps on the sides and in the corners. Since the dimming of the groups is separated, the load on any dimmer will never exceed $4 \times 50 \mathrm{~W}=200$ $\mathrm{W}$. Our specific dimmer has an operation range between $40 \mathrm{~W}-400 \mathrm{~W}$, TRIAC based phase-splitting power control. Bliss et al. [3] and Kim et al. [4] show solutions for this purpose, where they combine LEDs with solar halogens.

For LED units, the goal is to control the brightness of each individual color groups. To ensure the feasibility of future fine tuning, higher power (5 W) LED units are installed. LED units of the same color are connected in series and are supplied by individual power supplies through individual power controllers. The actual LED matrix wiring plan is shown in Figure 4.

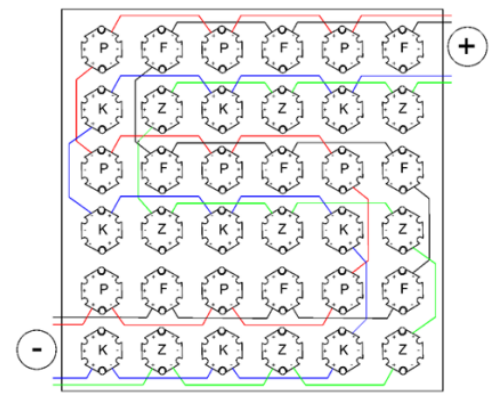

Figure 4

Positions and wiring of the LED units in the matrix

In case of LED lighting, it is essential to keep the amperage and voltage limit, which - in the case of these $3 \mathrm{~W}$ LED units - is $700 \mathrm{~mA}$ and $4-4.5 \mathrm{~V}$. Given these parameters, the color groups of the LED matrix are powered through a combination of four dimmable current sources and power regulators of the same type as the halogen lamps connected to the AC side. This design is capable of supplying the system with the maximal voltage supply, and can dim the light intensity through a phase-splitting based controller.

To ensure the proper operation conditions of the high-performance LED units, the heat dissipation of the device must be absorbed somehow, which in practice, is most often solved by installing a heatsink. In our case the heat sink must fulfil two roles; first, it must have the appropriate heat transfer properties to be able to dissipate the generated heat. Second, it must have a large enough useful surface area that it can hold the designed LED matrix $[1-4,12]$. At the beginning of the 
design process of the heat dissipation system, we suspect that heat sinks with an affordable price will not be able to provide the necessary heat dissipation rate, hence we decided to incorporate a fan. Further calculations are done assuming the presence of forced convection. Geometric dimensioning is done in an inverse method, meaning that some heat sink and fan combinations are selected, and then checked individually. After that, we choose the one that is most suitable for our demands. From here on out, the verification of the chosen heatsink - fan combination will be unfolded. The material of the chosen heat sink is aluminum, its bounding size is $190 \mathrm{~mm} \times 190 \mathrm{~mm} \times 50 \mathrm{~mm}$ [length $\mathrm{x}$ width $\mathrm{x}$ height]. Active cooling is provided by a fan with a diameter of $140 \mathrm{~mm}$. The nominal airflow rate of the fan is $126 \mathrm{~m}^{3} / \mathrm{h}$. The first step of the verification is to determine the thermal resistance of the heatsink given a certain airflow rate, which is calculated in an online program called My Heat Sink- Thermal Resistance Calculator [5]. After specifying the geometrical dimensions and the material of the heatsink, we get the thermal resistance as the function of the airflow rate.

According to relevant literature, the semiconductor material of the LED lights will remain properly operable below $125^{\circ} \mathrm{C}[7,15,22]$. In our case, the chosen heat sink - fan combination guarantees the proper operation of the LED units even at maximum loads the temperature is $88.9^{\circ} \mathrm{C}[5,12]$.

The last step is to provide the power supply of the cooling fan. The selected fan requires $12 \mathrm{VDC}$ to operate, which is provided by a $12 \mathrm{~V}, 2 \mathrm{~A}, 24 \mathrm{~W}$ industrial power supply unit. The entire power supply wiring of the sun simulator is shown in Figure 5.

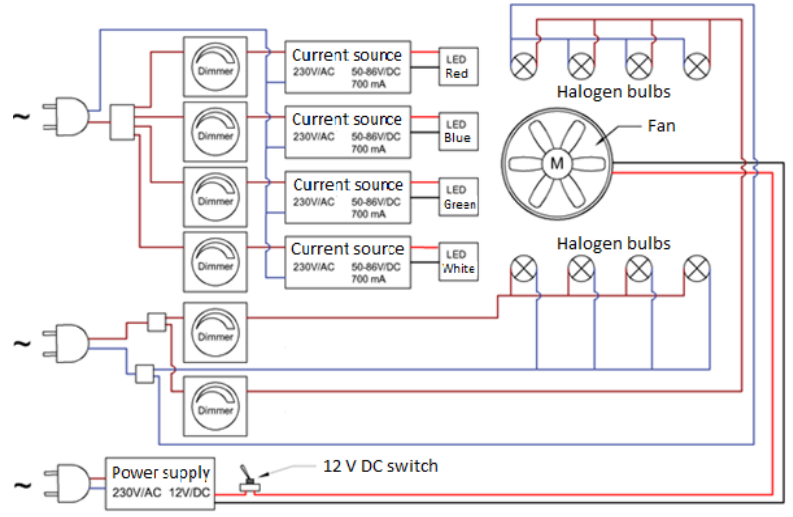

Figure 5

The power supply wiring of the sun simulator

\subsection{Framework}

The following main aspects are considered when designing the framework: 
- proper holder for the heatsink with the LED matrix

- stable halogen framework that can provide positional adjustability

- no interference with the light sources (by casting shadows)

The material of choice is an iron hollow section with a cross section of $20 \mathrm{~mm} \mathrm{x}$ $20 \mathrm{~mm}$, which is easy to weld, has high bearing capacity and is since quite stable. It is not liable to warping or deformation, and besides can be used to construct a relatively slim structure. Halogen bulbs are held by threaded stems attached to the grooves of radially placed grooved flat iron rods [17-19]. The heatsink hangs from four threaded iron balls. Above located the support bracket of fan providing the active cooling is. The horizontal, " $\mathrm{H}$ " shaped part, to which all the other elements mentioned so far connect, can be considered the central unit of the structure. The vertical elevation of said unit is provided by four feet, while the horizontal crosses on the feet are required to achieve high stability, since the center of mass is located comparatively high. The inspection range is located below the heatsink of the LED matrix [12]. The height of the inspection range is planned to be adjustable. The bounding size of the framework is $600 \mathrm{~mm}$ x $550 \mathrm{~mm}$. The visual representation of the completed framework is shown in Figure 6.

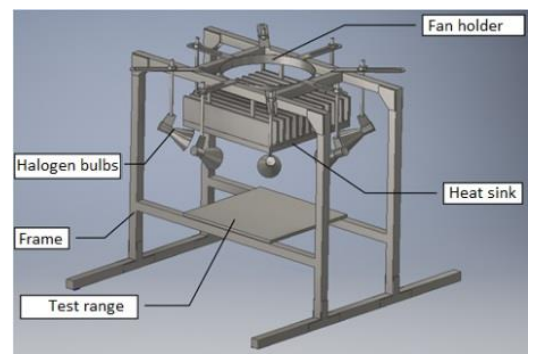

Figure 6

The designed construction of the sun simulator, with the main elements indicated

\subsection{The Realized Construction}

The Realized Construction can be seen in Figure 7. A two-channel laboratory power source with two current limiters are used to power the LED matrix, as shown in Figure 8.

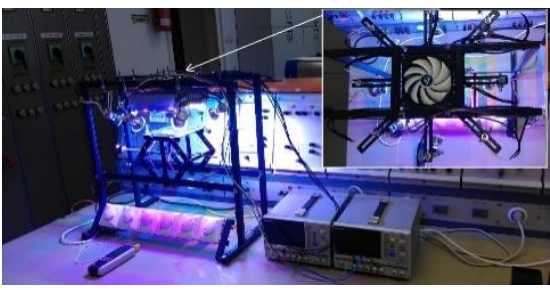

Figure 7

The complete sun simulator 


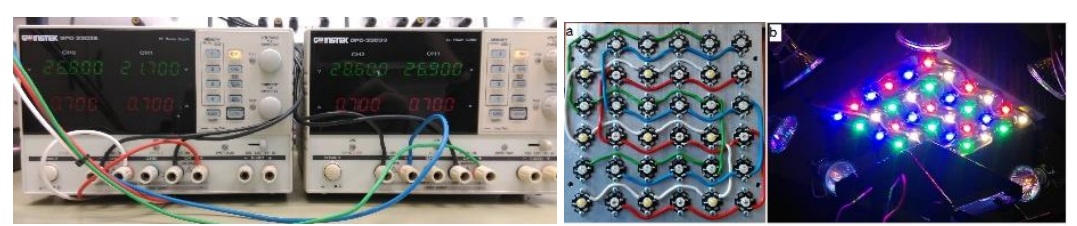

Figure 8

Power supplies used for the validation measurements and (a) Wiring of the LED matrix, (b) LED matrix during operation

\section{Validation of the Design}

This chapter is bound to describe the measurement of the previously calculated values (spectral structure, light intensity distribution, temperature of the LED), hereby describing the validation process of the sun simulator $[12,13]$.

\subsection{Inspection of Light Intensity Distribution}

An important quality of standard sunlight simulators is the magnitude and the distribution of the light intensity measured in the illuminated range. A light intensity measurement is performed on the finished construction. The inspection range - a square with width and depth of $15 \mathrm{~cm}$ - is split up to $1 \mathrm{~cm}^{2}$ segments, then the light intensity is measured by a handheld light intensity $\left[\mathrm{W} / \mathrm{m}^{2}\right]$ meter in every segment [20]. Measurements are done as described in Figure 9. As opposed to pre-measurements, this time the illumination is provided by the sun simulator with the previously defined fixed and design parameters. The resulting light intensity distribution is shown in Figure 10.

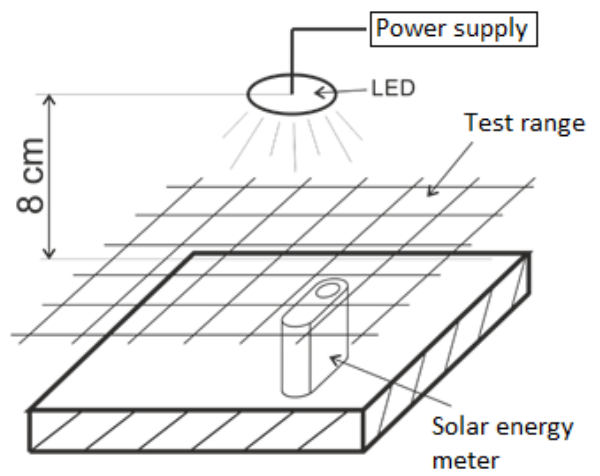

Figure 9

Measurement setup for measuring light intensity distribution 
By examining the measured values, it can be deducted that light intensity values are within a certain range, depending on the measuring position. The smallest measured intensity value is $\operatorname{Imin}=868 \mathrm{~W} / \mathrm{m}^{2}$ and the largest is $\operatorname{Imax}=1,060$ $\mathrm{W} / \mathrm{m}^{2}$. The arithmetic mean of the measured light intensity values is $951 \mathrm{~W} / \mathrm{m}^{2}$. It can clearly be seen that the light intensity distribution is relatively homogenic within the inspection range. Inhomogeneity can be calculated by a standardized equation (3) [20].

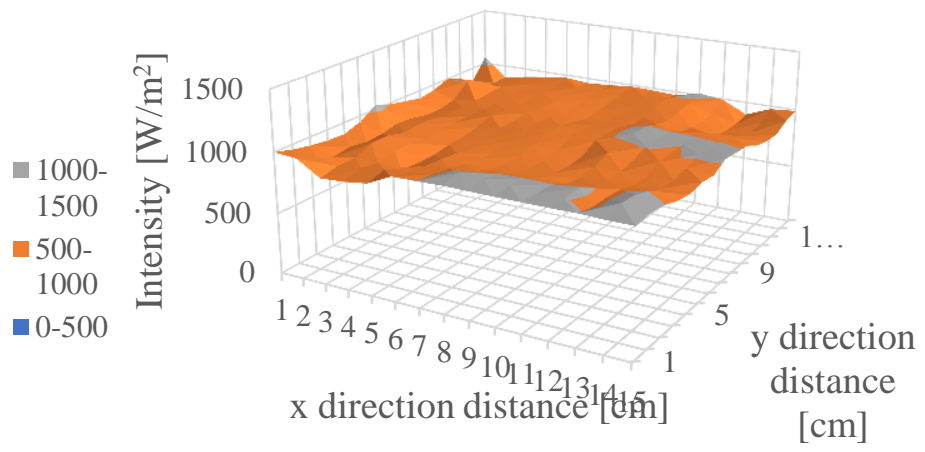

Figure 10

Measured light intensity distribution within the inspection range

According to the standard, for class $\mathrm{C}$ class sun simulators, the inhomogeneity of the light intensity distribution may not be more than $10 \%$. The light intensity distribution of our sun simulator has a $9.96 \%$ inhomogeneity, which means the device complies with the standard.

\subsection{Inspection of Spectral Structure}

To check the spectral structure of the illumination, an Ocean Optics USB 4000 type spectrometer is used. Due to the high directional dependence of the device, a white sheet of paper is placed atop the inspection range, and the probe is fixed in a position facing the scattered light. The measurement environment is shown in Figure 11. 


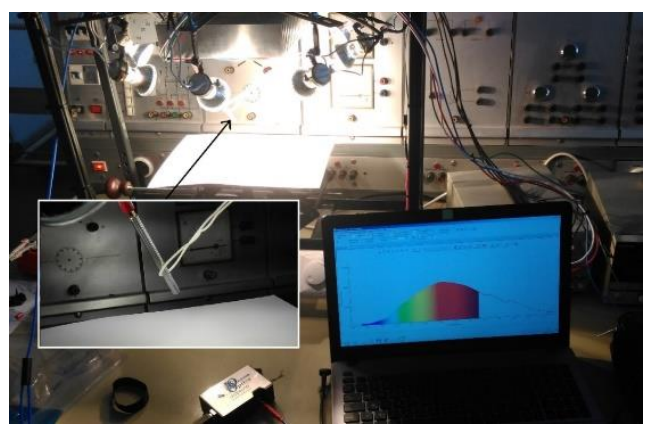

Figure 11

Spectral structure measurement

During the spectrum analysis, the spectrum structure of the finished sun simulator is examined. The applied spectrometer is not suitable to perform absolute light intensity measurements; however, it allows for the spectral curve of the sun simulator and natural sunlight to be recorded. Thus, it is possible to compare the spectrum structure of the sun simulator and natural sunlight, and calculate their spectral conformity $[5-8,20]$. The measured spectral curves of the sun simulator and natural sunlight are shown in Figure 12. The same tendency can be seen in some similar publications, such as Kádár et al [8]. and Kenny et al. [20].

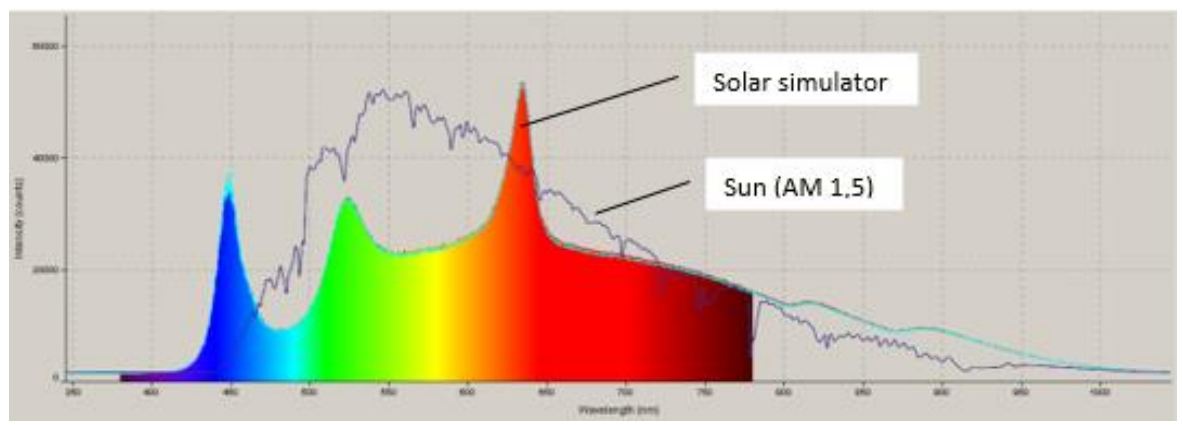

Figure 12

Spectral structure of the sun simulator and natural sunlight

Table 6

Actual spectral conformity within the inspected wavelength ranges [5-8]

\begin{tabular}{|c|c|c|c|c|c|c|}
\hline $\begin{array}{c}\text { Wavelength range } \\
{[\mu \mathrm{m}]}\end{array}$ & $0.4-0.5$ & $0.5-0.6$ & $0.6-0.7$ & $0.7-0.8$ & $0.8-0.9$ & $0.9-1.1$ \\
\hline $\begin{array}{c}\text { Spectral } \\
\text { conformity [\%] }\end{array}$ & 120.9 & 59.0 & 89.3 & 116.8 & 160.6 & 182.7 \\
\hline
\end{tabular}


The rate of the measured spectral conformity is always within the $40 \%-200 \%$ range assigned to a class $\mathrm{C}$ sun simulator, hence the desired spectral structure can be provided [10, 11]. The unexpected correctness of the lowest wavelength interval is caused by a slight shift in the intensity peak of the blue LED lights, therefore the application of UV dominant LED units may not be necessary. Many other researchers received similar results, inter alia, Kenny et al. [20] and Hussain et al. [25].

\subsection{Inspection of Operating Temperature}

During the operation of the sun simulator, heat production caused by current running through certain units is inevitable. Excessive warming may result in the deterioration of efficiency, it can damage the device, and may result in fire- and accident hazards, hence it is important to determine if the operating temperatures of the device fall within the safe zone [15].

Three thermometer probes are placed at three points of the - initially roomtemperature - device, the operating temperatures will be measured and inspected in these points. One sensor is placed atop a solar cell above the inspection range, another is placed on the surface of the heatsink below the LEDs, and the last one is on the back of the heatsink. The heatsink is cooled by a fan above it. During the measurement, the halogen lamps are operated at maximum load, the LED units are uniformly powered at $700 \mathrm{~mA}$. A four-channel digital thermometer is used to measure and save the results once every second. The whole measurement is carried out in roughly 20 minutes, at which point constant temperature values are obtained at all three points [15]. The obtained results are shown in Figure 13.

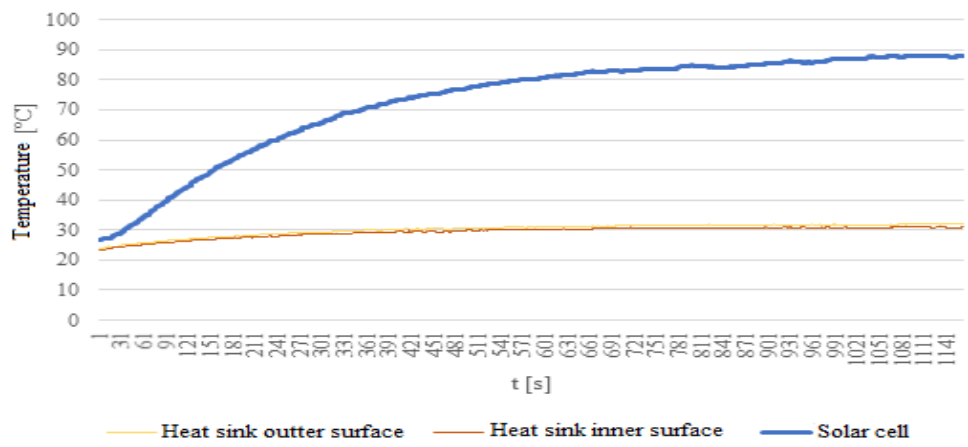

Figure 13

Temperature transient of the surface and the back of the heatsink

Even at the critical points of the device no dangerous overheating is experienced. After 20 minutes of operation, the temperature stabilizes at a constant value on both sides of the heatsink, which in both cases are around $30^{\circ} \mathrm{C}$. Measuring the temperature of the semiconductor material of the LED is not possible, thus the 
dimensioning of the heatsink is not executable. However, the temperature increase on the heatsink is barely $10{ }^{\circ} \mathrm{C}$, which is well within the generally accepted $20^{\circ} \mathrm{C}$ boundary, meaning that the cooling is without doubt applicable.

As expected, the temperature of the solar cell is much higher than the temperature measured on the heatsink, since it is being illuminated by 8 halogen lamps and 36 LED units simultaneously. The temperature of the solar cell stabilized at around $88{ }^{\circ} \mathrm{C}$. This is roughly the same temperature as it would be of a solar cell illuminated by natural sunlight on a sunny summer day. In the light of this information it can be stated that the solar cell will not suffer any damages from this amount of heat, although its temperature, according to plans, is going to be much lower. In conclusion, the sun simulator can be declared safely operable in terms of heating.

\section{Results and Conclusions}

Herein, we provide an overview of the whole work, in which, the design and construction of the standard sun simulator was executed. During the design, fixed parameters and, with the help of optimizing calculations, variable parameters were defined, while always keeping the proper light intensity distribution and the spectral structure of the illumination. Then the sun simulator was assembled, and test measurements were implemented.

The light intensity distribution was inspected, using a light intensity meter, in cases with different halogen lamp positions. We achieved an inhomogeneity of $9.96 \%$, which is acceptable for a standard class C sun simulator. The average of the light intensity in this case is $951 \mathrm{~W} / \mathrm{m}^{2}$, which is slightly below the 1,000 $\mathrm{W} / \mathrm{m}^{2}$ peak, but is a good approximation of an average light intensity on a clear day, hence it is applicable to inspect solar cells.

The spectral composition of the illumination was inspected using a spectrometer. According to the design, proper spectral conformity was expected in each interval but one; between the wavelengths of $400 \mathrm{~nm}-500 \mathrm{~nm}$. In possession of the measurement results, it can be said that the spectral conformity of the sun simulator is within the prescribed class $\mathrm{C}$ standards, meaning that the methods used during the design of the sun simulator were correct and are applicable to provide close to reality calculations.

An important aspect when designing the sun simulator is to keep the costs realistic. For small sized solar cells, a standard illumination can be provided by a relatively inexpensive device; the full price of this construction is 600 Euro.

\section{Acknowledgement}

"The described article was carried out as part of the EFOP-3.6.1-16-2016-00011 "Younger and Renewing University - Innovative Knowledge City - institutional development of the University of Miskolc aiming at intelligent specialization" project implemented in the framework of the Szechenyi 2020 program. The 
realization of this project is supported by the European Union, co-financed by the European Social Fund."

\section{References}

[1] Wang, W.: Simulate a 'Sun' for Solar Research: A Literature Review of Solar Simulator Technology, Royal Institute of Technology, Department of Energy Technology, Swede, Stockholm, 2014, p. 7

[2] Kohraku, S., Kurakowa, K.: A Fundamental Experiment for DiscreteWavelenght LED Solar Simulator, Solar Energy Materials \& Solar Cells 90. 2006, pp. 3364-3370

[3] Bliss, M., Betts, R., T., Gottschlag, R.: An LED-based photovoltaic measurement system with variable spectrum and flash speed, Solar Energy Materials \& Solar Cells 93, 2009, pp. 825-830

[4] Kim, A, K., Dostart, N., Huynh, J., Krein, T., P.: Low-Cost Solar Simulator Design for Multi-Junction Solar Cells in Space Applications, Power and Energy Conference at Illinois, 2014, p. 6

[5] Bodnár, I.: (2018) Electric parameters determination of solar panel by numeric simulations and laboratory measurements during temperature transient, Acta Polytechnica Hungarica, Vol. 15, No. 4, 2018, pp. 59-82

[6] Kativar, M., Balkom, M., Rindt, C. C. M., Keizer, C., Zondag, H. A.: Numerical model for the thermal yield estimation of unglazed photovoltaicthermal collectors using indoor solar simulator testing, Solar Energy, Vol. 155, No. 10, 2017, pp. 903-919

[7] Tawfik, M., Tonnellier, X., Sansom, C.: Light source selection for a solar simulator for thermal applications: A review, Renewable and Sustainable Energy Reviews, Vol. 90, 2018, pp. 802-813

[8] Kádár, P., Varga, A.: Measurement of spectral sensitivity of PV cells. 2012 IEEE $10^{\text {th }}$ Jubilee International Symposium on Intelligent Systems and Informatics. 2012, pp. 549-552

[9] Chegaar, M., Ouennoughi, Z., Hoffmann, A.: A new method for evaluating illuminated solar cell parameters, Solid-State Electronic, Vol. 45, No. 2, 2001, pp. 293-296

[10] Varga, A., Libor, J., Rácz, E., Kádár, P.: A small laboratory-scale experimental method and arrangement for investigating wavelength dependent irradiation of solar cells, Proceedings of the $11^{\text {th }}$ IEEE International Symposium on Applied Computational Intelligence and Informatics, 2014, pp. 137-141

[11] Munoz-Garcia, M. A., Marin, O., Alonso-García, M. C., Chenlo, F.: Characterization of thin film PV modules under standard test conditions: 
Results of indoor and outdoor measuremnets and the effects of sunlinght exposure, Solar Energy, Vol. 86, No. 10, 2012, pp. 3049-3056

[12] González, M. I.: An LED solar simulator for student labs, Physics Education, Vol. 52, No. 3, 2017, p. 5

[13] Sowmy, D. S., Ara, P. J. S., Prado, R. T. A.: Uncertainties associated with solar collector efficiency test using an artificial solar simulator, Renewable Energy, Vol. 108, 2017, pp. 644-651

[14] Siddiqui, R., Kumar, R., Jha, K. G., Morampudi, M., Rajput, P., Lata, S., Agariya, S., Nanda, G., Raghava, S. S.: Comparison of different technologies for solar PV (Photovoltaic) outdoor performance using indoor accelerated aging tests for long term reliability, Energy, Vol. 107, No. 15, 2016, pp. 550-561

[15] Gu, R., Ding, J., Wang, Y., Yuan, Q., Wang, W., Lu, J.: Heat transfer and storage performance of steam methane reforming in tubular reactor with focused solar simulator, Applied Energy, Vol. 233-234, 2019, pp. 789-801

[16] Moss, R. W., Shire, G. S. F., Eames P., Henshall P. Hyde T., Arya F.: Design and commissioning of a virtual image solar simulator for testing thermal collectors, Solar Energy, Vol. 159, 2018, pp. 234-242

[17] Boubault, A., Yellowhair, J., Ho, C. K.: Design and Characterization of a $7.2 \mathrm{~kW}$ Solar Simulator, Journal of Solar Energy Engineering, Vol. 139, No. 3, 2017, p. 8

[18] Esen, V., Saglam, S., Oral, B.: Light sources of solar simulators for photovoltaic devices: A review, Renewable and Sustainable Energy Reviews, Vol. 77, 2017, pp. 1240-1250

[19] Wang, W., Aichmayer, L., Garrido, J., Laumert, B.: Development of a Fresnel lens based high-flux solar simulator, Solar Energy, Vol. 144, 2017, pp. 436-444

[20] Kenny, R. P., Vigan, D., Salis, E., Bardizza, G., Norton, M., Müllejans, H., Zaaiman, $\mathrm{W} .:$ Power rating of photovoltaic modules including validation of procedures to implement IEC 61853- 1 on solar simulators and under natural sunlight, Paper Presented at 27TH EU PVSEC 2012, Frankfurt, Germany, Photovoltaics, Vol. 21, No. 6, 2013, pp. 1384-1399

[21] Jiang, H., Lu, L., Sun, K.: Experimental investigation of the impact of airborne dust deposition on the performance of solar photovoltaic (PV) modules, Atmospheric Environment, Vol. 45, No. 25, 2011, pp. 4299-4304

[22] Chen, F., Wittkopf, S. K., Ng, P. K., Du, H.: Solar heat gain coefficient measurement of semi-transparent photovoltaic modules with indoor calorimetric hot box and solar simulator, Energy and Buildings, Vol. 53, 2012, pp. 74-84 
[23] Hamadani, B. H., Chua, K., Roller, J., Bennahmias, M. J., Campbell, B., Yoon, H. W., Dougherty, B.: Towards realization of a large- area lightemitting diode- based solar simulator, Photovoltaics, Vol. 21, No. 4, 2013, pp. 779-789

[24] Guo, P., Wang, Y., Meng, Q., Li, J.: Experimental study on an indoor scale solar chimney setup in an artificial environment simulation laboratory, Applied Thermal Engineering, Vol. 107, 2016, pp. 818-826

[25] Hussain, F., Othman, M. Y. H., Yatim, B., Ruslan, H., Sopian, K., Anaur, Z., Khairuddin, S.: Fabrication and Irradiance Mapping of a Low Cost Solar Simulator for Indoor Testing of Solar Collector, Journal of Solar Energy Engineering, Vol. 133, No. 4, 2011, p. 4

[26] Precup, R. E., Preitl, S.: Stability and Sensitivity Analysis of Fuzzy Control Systems, Mechatronics Applications, Acta Polytechnica Hungarica, Vol. 3, No. 1, 2006, pp. 61-76

[27] Pozna, C., Precup, R. E., Tar, J. K., Skrjank, I., Preitl, S.: New results in modelling derived from Bayesian filtering, Knowledge-Based Systems, Vol. 23, No. 2, 2010, pp. 182-194

[28] Ürmös, A., Farkas, Z., Farkas, M., Sándor, T., Kóczy, L. T., Nemcsics, Á.: Application of self-organizing maps for technological support of droplet epitaxy, Acta Polytechnica Hungarica, Vol. 14, No. 4, 2017, pp. 207-224

[29] Vrkalovic, S., Lunca, E. C., Borlea, J. D.: Model-free sliding mode and fuzzy controllers for reverse osmosis desalination plants, International Journal of Artificial Intelligence, Vol. 16, No. 2, 2018, pp. 208-222 